\title{
COVD-19 And Final Year Medical Students' Learning Process In Oman: Exploring Knowledge, Consequences And Preparedness For Internship
}

\author{
Hatem Al-Saadi, Fatma Al-Busaidi, Marwa Al-Kalbani, Said Al-Abri, Haya Malallah, \\ Jameela Al-Saadi, Ahlam Al-Saadi, Vittal Rao, and Tariq Dhiyab Al-Saadi
}

\begin{abstract}
Background: Coronavirus Disease 2019 (COVID19) is a cluster of acute respiratory illness that was first identified in Wuhan Province in China. The virus causing this syndrome is known as SARS-CoV-2. The COVID 19 pandemic affects every aspect of our life, including education, work, travel, business, and social life. Aim: To evaluate the final year medical students' perceptions of their preparedness for internship and assess their awareness of COVID-19 and patients' management in the Sultanate of Oman.

Methods: A cross-sectional survey of cohort of final year medical students at Sultan Qaboos University (SQU) and National University of Science and Technology (NU) was distributed to 227 students in both medical schools from 21st to 28th of April 2020. A total of 99 students fully completed the survey with a response rate of $43.6 \%$. The survey had five sections exploring perceptions on preparedness for internship and awareness and knowledge on COVID-19, training, and implications.

Results: Majority of participants $(\mathbf{8 2 . 8 \%})$ were $24-26$ years old. Omani students accounted for $88.9 \%$ of the participants. 81.4 of SQU participants were competent in carrying ward's practical procedures comparing to $39.3 \%$ of $\mathrm{NU}$ participants $(p<0.05)$. There was a higher level of awareness of the current ministry of health policies on the management of patients with COVID-19 with NU participants than SQU (p < 0.005). Training on preparedness for COVID-19 was higher in females than males $(p=0.02)$.

Conclusion: COVID-19 has affected the teaching process and limited student- patient interactions. With ongoing rise in confirmed cases every day, the uncertainty of the duration of the suspension of on-campus teaching in higher education institutions in Oman is still ongoing. The overall perceptions of preparedness for internship among medical students in Oman are good. However, further education in emergency management and training on practical procedures are still required.
\end{abstract}

Published on June 10, 2020

Hatem Al-Saadi, North Midlands Institute of Metabolic and Bariatric Surgery, Stafford, United Kingdom; Gulf Research Collaboration Group (GRCG).

Fatma Al-Busaidi, College of Medicine \& Health Science, National University of Science \& Technology, Sultanate of Oman.

Marwa Al-Kalbani, Ministry of Health, Sultanate of Oman; Oman Medical Specialty Board, Ibri Hospital, Sultanate of Oman.

Said Al-Abri, Statistical Researcher, Center for Government Communications, General Secretariat to the Cabinet, Muscat, Sultanate of Oman.

Haya Malallah, Gulf Research Collaboration Group (GRCG), Kuwait.

Jameela Al-Saadi, Ministry of Health, Muscat, Sultanate of Oman.

Ahlam Al-Saadi, Gulf Research Collaboration Group (GRCG), Oman.

Vittal Rao, North Midlands Institute of Metabolic and Bariatric Surgery, Stafford, United Kingdom.

Tariq Dhiyab Al-Saadi, Department of Neurology and Neurosurgery, Montreal Neurological Institute - McGill University, Canada.

(corresponding e-mail: t.dhiyab@ ${ }^{\circledR}$ otmail.com).
Index Terms - coronavirus, COVID-19, medical students, final year, preparedness, internship, Oman, Medical education.

\section{INTRODUCTION}

Coronavirus Disease 2019 (COVID-19) is a cluster of acute respiratory illness that was first identified in Wuhan Province in China. The virus causing this syndrome is known as SARS-CoV-2 [1]. The World Health Organization (WHO) declared the outbreak as a public health emergency of international concern on 30th of January 2020 and on the 11th of March it was announced that the COVID-19 outbreak is a global pandemic [2].

The COVID 19 pandemic affects every aspect of our life, including education, work, travel, business, and social life. This goes from suspending all forms of education, reducing the number of workforce to minimum in different working areas, making people to work online at home, physical distancing and social exclusion, restriction of travel, health system crisis and economic decline are some issues resulting from facing COVID-19 [3].

The COVID-19 pandemic is putting an enormous strain on the health care system. This is due in part to the rapid increase in the number of cases and in part because health care professionals are themselves getting sick - in some cases fatally succumbing to the virus [4]. Moreover, it impacts on medical education in general and the training and preparation of final year medical students in particular. Students are not able to complete their clinical placements in an effective and safe environment because of the lockdown caused by the pandemic. Transferring medical education into online platform results in loss of in hospital clinical rotations, simulation laboratory and interactive medical school's sessions. Thus, limiting the process of preparing and equipping these students, who will soon be practicing in hospitals, for real life medical emergencies [4].

As final-year medical students are approaching the end of the academic year, in few months, they will be joining the medical forces in different hospitals and practicing as interns/ supervised doctors. Students entering the practice increase the medical workforce which is crucial for the increasing demands in facing current pandemic. This helps the health system to cope with the increasing number of patients, speeding up post-pandemic recovery, maintaining the essential medical services, and providing safe society to prevent disease transmission. Effective human resources are essential to ensure adequate staff capacity and the continuity of the main operations [5]. 
There are two medical schools in Oman: one public, Sultan Qaboos University (SQU), and one private, National University of Science and Technology (NU). The internship is a term used in medicine for the graduates who attained their medical degree and still not licensed to work without supervision. It is a transition of the medical students to doctors [6]. In Oman, the internship is a one-year period divided into three 4-months rotations. The new graduates will be able to join various departments in different hospitals in the coming few months. Interns will transform their medical school knowledge into practice which will equip them with skills to approach and manage patients under the supervision of senior doctors. The internship period is very crucial to strengthen intern's clinical skills and helps them learn the professional attitudes and behaviors [7]. It also helps to produce a capable and independent doctor for future practice.

The new graduates join the medical workforce with substantial knowledge and clinical experience, yet preparation and guidance provided varies by institution. The literature found that inadequate career preparation, either due to inadequate institutional guidance or level of awareness, is one of the key stressors for medical students [8].

This cross-sectional study aims to evaluate the final year medical students' perceptions on their preparedness for internship and assess their knowledge on COVID-19 and patients' management. It also extends to illustrate COVID19 specific training and explore the pandemic consequences on the medical education in the Sultanate of Oman.

\section{MATERIALS AND METHODS}

\section{A. Participants and Setting}

A cross-sectional study of cohort of final year medical students at Sultan Qaboos University (SQU) and National University of Science and Technology (NU) was conducted with distribution of electronic survey. The electronic survey was sent to all final year medical students in both medical schools $(n=227)$ and survey fieldwork ran from 21 st to 28th of April 2020. A total of 99 students fully completed the survey with response rate of $43.6 \%$.

Inclusion criteria were final year medical students. Exclusion criteria were students not in final year, abroad studying students and incomplete responses.

\section{B. Questionnaire Design}

A structured self-administered questionnaire consisting of five sections was distributed electronically to all potential participants in their final medical year. The survey five sections had close ended questions and included:

a) socio-demographic questions (6 questions);

b) perceptions on preparedness for internship (8 questions);

c) awareness and knowledge on COVID-19 (10 questions);

d) COVID-19 training (4 questions), and e) COVID-19 consequences and implication (6 questions).

Awareness questions were based on the interim guidance and information for healthcare workers provided by Centre of Disease Control (CDC), and Coronavirus disease
(COVID-19) outbreak: rights, roles and responsibilities of health workers published by WHO $[9,10]$.

The survey elements were developed according to the objectives and variables of the study. All survey questions, except demographics, were closed questions of either 2scales (Yes or No) or 3-scales (True, False or I do not Know). Content validity of the survey was checked and critiqued by group of experts in medical education. The survey was piloted on 10 subjects prior to conducting the study. Participants' consent to participate in the study was given through return of the completed questionnaire.

In order to exclude participants not in final year, expected year of graduation was added to the demographic part. Fig. 1 shows the flow diagram for the inclusion and exclusion of responses.

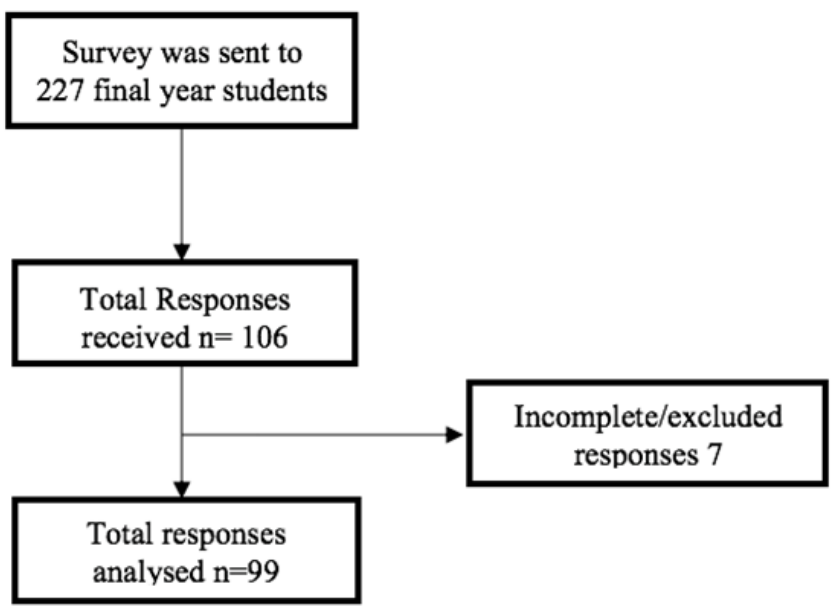

Fig. 1. Flow diagram for inclusion and exclusion of responses.

\section{Statistical analysis}

Collected data were tubulated in Excel and statistical analysis was carried out using Statistical Package for the Social Sciences (SPSS v21 Inc, Chicago, Illinois, USA). Numbers and percentages were used to represent categorical variables data. Continuous variable data were presented as mean and standard deviation. Associations and significance differences between variables were tested using Fisher's Extract Test and Chi square test. A p-value of $<0.05$ was considered statistically significant.

\section{RESULTS}

\section{A. Demographics}

Total completed responses received that met the inclusion criteria of the study were 99 (84 females, 15 males). Majority of participants $(82.8 \%)$ were 24-26 years old. Omani students accounted for $88.9 \%$ of the participants. Four of the participants are intending to do their internship program abroad. Participants' demographics are shown in Table I. 
TABLE I: DEMOGRAPHIC INFORMATION OF PARTICIPANTS

\begin{tabular}{lc}
\hline \hline \multicolumn{1}{c}{ Characteristics } & Percentage (n) \\
\hline Age: & \\
$21-23$ & $15.2 \%(15)$ \\
$24-26$ & $82.8 \%(82)$ \\
$27-29$ & $2.0 \%(2)$ \\
\hline Gender: & \\
Male & $15.02 \%(15)$ \\
Female & $84.8 \%(15)$ \\
\hline Nationality: & \\
Omani & $88.9 \%(88)$ \\
Non-Omani & $11.1 \%(11)$ \\
\hline University: & \\
National University of Science and & $56.6 \%(56)$ \\
Technology & $43.4 \%(43)$ \\
Sultan Qaboos University & $100 \%(99)$ \\
\hline Total
\end{tabular}

\section{B. Perceptions on Preparedness for Internship}

Eight closed ended questions were used to evaluate participants' perceptions on their preparedness for internship. In four questions (B1, B3, B5 and B8), more than 90 participants answered there were competent in the specific questions. Lower numbers of competency were found in questions related to emergency management of ill patients $(B 6 n=49)$ followed by confidence in carrying ward procedures $(\mathrm{B} 4, \mathrm{n}=57)$. Participants responses are shown in Fig. 2.

The study found that $81.4 \%$ of SQU participants were able to carry basic ward procedures (e.g. cannulation and NG-Tube), while only $39.3 \%$ of the NU students answered they were competent with these procedures. The difference in the competency in carrying basic ward procedures between the 2 medical schools was statistically significant $(\mathrm{p}<0.001)$. The competency with principles of infection control and the ability to take part in ACLS in emergency situation were also significant between the two medical schools $(\mathrm{p}<0.001)$.

\section{Covid-19 Awareness}

To evaluate the level of awareness of the participants, 10 knowledge-based closed-ended questions were asked (Table II). Questions were then categorized into five themes (Pathogenesis, WHO role, Health Measures, Medical Managements, and Infection Control). Correct answered per theme were then compared to identify gaps in knowledge as shown in the graph (Fig. 3).

TABLE II: 1 THEMES OF KNOWLEDGE BASED QUESTIONS

\begin{tabular}{|c|c|}
\hline Theme s & Questions \\
\hline \multirow{4}{*}{ Pathogenesis } & Coronaviruses are a newly discovered family of viruses \\
\hline & Molecular tests, using Polymerase Chain Reaction (PCR) \\
\hline & have been developed and are being used to detect the \\
\hline & SARS-CoV-2 virus causing COVID-19 \\
\hline \multirow[b]{2}{*}{ WHO role } & $\begin{array}{l}\text { The response to COVID-19 is entirely dependent on the } \\
\text { World Health }\end{array}$ \\
\hline & $\begin{array}{l}\text { Mental health and psychosocial support are not an } \\
\text { important part of emergency planning and response } \\
\text { measures in outbreak }\end{array}$ \\
\hline \multirow[b]{2}{*}{$\begin{array}{l}\text { Health } \\
\text { measure }\end{array}$} & $\begin{array}{l}\text { Syndromic screening aims to detect people in the } \\
\text { incubation period }\end{array}$ \\
\hline & $\begin{array}{l}\text { The incubation period is the time from exposure to the } \\
\text { causative agent until the first symptoms develop and is } \\
\text { characteristic for each disease agent }\end{array}$ \\
\hline \multirow{2}{*}{$\begin{array}{l}\text { Medical } \\
\text { management }\end{array}$} & $\begin{array}{l}\text { For the management of COVID-19 in hospital, the flow } \\
\text { and transfer of patients should be planned ahead }\end{array}$ \\
\hline & $\begin{array}{l}\text { Existing medicines for other diseases may be able to be } \\
\text { re-purposed for COVID- } 19\end{array}$ \\
\hline \multirow{2}{*}{$\begin{array}{l}\text { Infection } \\
\text { control }\end{array}$} & $\begin{array}{l}\text { Surgical mask is enough personal protective equipment } \\
\text { for transferring asymptomatic positive patients with } \\
\text { covid-19 }\end{array}$ \\
\hline & $\begin{array}{l}\text { Hand rub with soap and water for at least } 10 \text { second is the } \\
\text { preferred method for soiled hands }\end{array}$ \\
\hline
\end{tabular}

\section{B. Perceptions on Preparedness for internship}

B8 Aware of your limitations and approach senior staff for help when you feel uncertain

B7 Able to take part in advanced life support in emergency situatio

B6 Confident in early management of emergency with COVID-19 patient

B5 Aware of the principles of infection control

B4 Confident in carrying out basic ward procedure

B3 Capable of communicating effectively and sensitively with patient

B2 Competent to take responsibility of the care of the patien

B1 Competence in performing a comperhensive clinical assessment
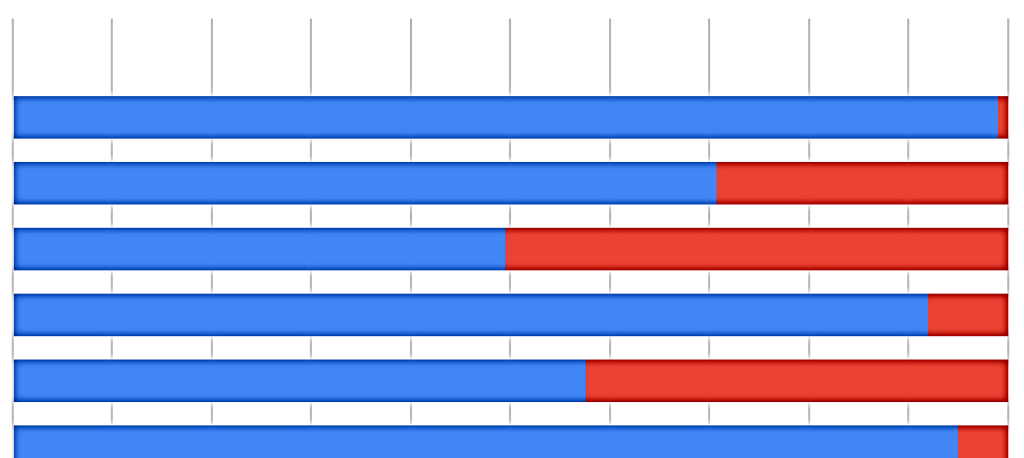

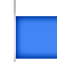

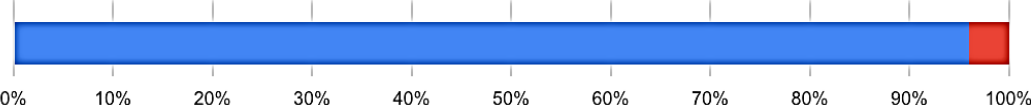

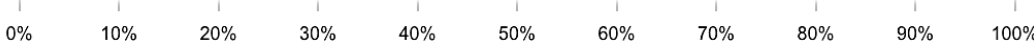

Fig. 2. Participants perceptions on their preparedness for internship. 


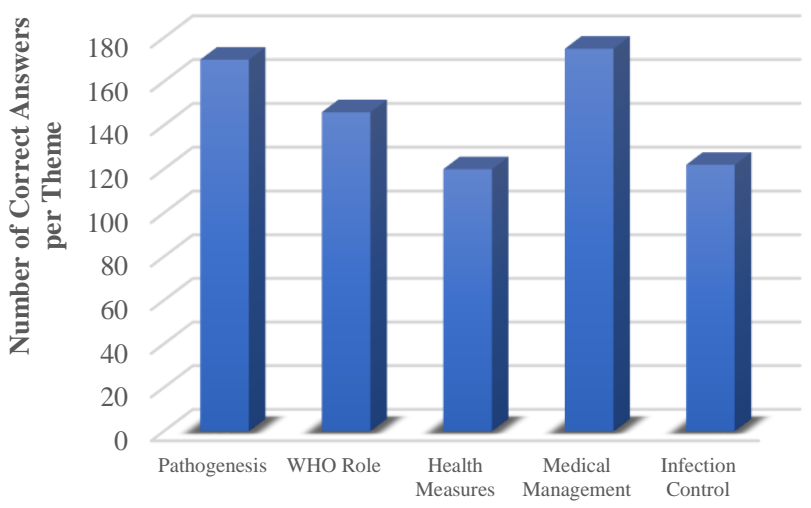

Fig. 3. COVID-19 Awareness: Number of correctly answered themes.

The number of correct answers were low in questions related to infection control followed by Health measures themes. Despite the overall satisfactory level of awareness (>50\% correct answers) for each theme, gaps in the knowledge have been identified when looking at questions individually. The question related to hand hygiene duration (C10) was the least correctly answered question (Correct 39, incorrect 57, I do not know 3). The overall of participants answers of the knowledge-based questions per medical school are shown in Fig. 4.

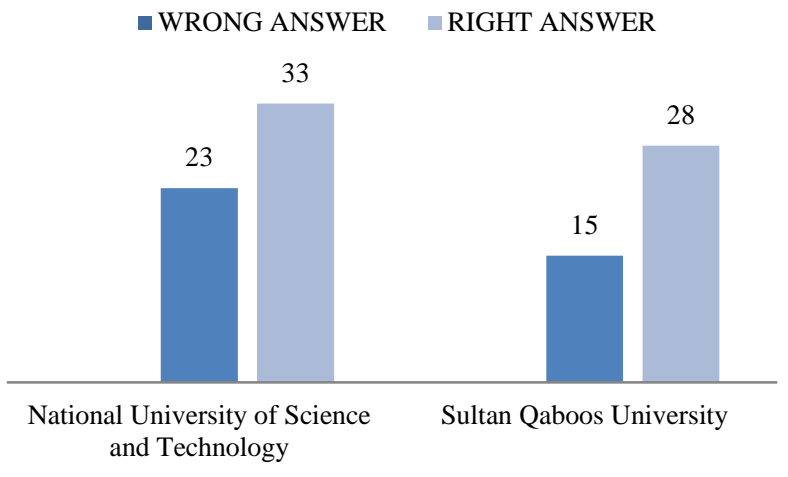

Fig. 4. Difference in awareness questions between the 2 medical schools.

\section{COVID-19 Training}

Training and latest guidelines and recommendations updates on COVID-19 for medical students were explored in this section. Majority of participants had training on hand hygiene in the last 12 months $(86.9 \%)$. However, only 18 participants had formal specific training about COVID-19 preparedness and management $(18.8 \%)$. About $12.1 \%$ of participants participated on online course or a form of teaching about COVID-19. There was higher level of awareness of current ministry of health policies on management of patients with COVID-19 among NU participants than SQU ( $\mathrm{p}<0.005)$. Training on preparedness for covid-19 was higher in female than male ( $\mathrm{p}=0.02)$.

\section{E. Covid-19 Consequences}

Along with other aspects of life, the pandemic of COVID19 also affects the education process. This section illustrates the implication of the pandemic on the teaching process. Participants from the 2 medical schools are currently off campus and continuing their college education online. With all participants, clinical rotations, examinations, and clinical clerkship have been affected by the pandemic. About one quarter of the participants have volunteered to help with the healthcare professionals during the pandemic. This was significant for different age groups $(p=0.04)$. The measures and preparedness in tackling the pandemic have illustrated the role of infection control and public health and increased participants knowledge on these as a specialty. Therefore, 47 participants have expressed their interest in pursuing Public Health and infection control as a career.

\section{DISCUSSION}

This prospective cross-sectional study explores final year medical students and COVID-19 perceptions on preparedness, awareness, training, and implications on education. The first two confirmed COVID-19 cases were diagnosed in the Sultanate were linked to travel and were diagnosed on the 23rd February 2020 [11]. To date, there are 8118 confirmed cases [12]. This wide spread of COVID19 has serios implications for public institutions and raised concerns for medical students. While final year medical students are preparing to take assessments and examinations, substantial number of medical schools across the globe has suspended clinical rotations and direct patient interactions. In countries like the United Kingdom [13], Kuwait and Ireland, several medical schools have announced the end of the academic year and expediated graduation to allow newly graduated doctors to join the medical force.

The study demonstrated overall high levels of perceptions on preparedness for internship by final year medical students in Oman. However, further training on carrying basic ward procedures (e.g. cannulation and NG-tube) is required. There is low perception of competency in early emergency management of patient with COVID-19. This indicates the necessity of further education and training in this matter as interns are part of the frontline dealing with patients with COVID-19.

The response to COVID-19 is dependent on the individual country emergency plan under the guidance of the WHO [5]. However, the study found mixed responses in perceptions were only $63 \%$ of participants agreed that the response to tackle the emergency is not entirely the role of the WHO but rather individual country. There is a good understanding on the incubation period of the SARS-CoV-2 virus. However, the study found low levels of understanding on syndromic screening which is the screening of travelers in the airport to prevent travel of symptomatic individuals. Based on the early data from China, the average incubation period for COVID-19 is five days [14].

The overall of correctly answered knowledge-based questions on COVID-19 awareness levels were different between the two medical schools with higher levels in SQU than NU students (65.1\% and $58.9 \%$ respectively). A study on COVID-19 awareness among healthcare students and professionals in Mumbai [15] showed a higher percentage of correct answers in medical students $(74.10 \%)$ than our overall findings (61.6\%). However, the finding of a survey of participants perceptions on knowledge on emerging infection in the Kingdom of Saudi Arabia showed that $61 \%$ 
of participants perceive their knowledge as low [16].

Hand hygiene is an effective way to prevent the spread of pathogens and infection in healthcare setting. The two basic techniques to clean hands are hand washing and hand rubbing. For healthcare setting, the $\mathrm{CDC}$ recommends the use of Alcohol Based Hand Rub (ABHR) with at least $60 \%$ ethanol or $70 \%$ isoproranol. The recommendation for visibly soiled hands is to be washed with soap and water for at least 20 seconds [17]. In this study, although 86 participants had training on hand hygiene in the last 12 months, only 38 participants correctly answered the infection control hand hygiene question. A recent study reported that only $51.9 \%$ of their respondents were aware one the preferred method of hand hygiene in health care settings [18].

Personal protective equipment (PPE) plays a particularly important role in the prevention of transmission of the COVID 19 infection. PPE needed for patients with confirmed and possible COVID infection and needed for the health care workers when caring for suspected or confirm cases of COVID-19 [19]. PPE indicated for use in COVID19 include face mask, eye protection like goggles or disposable face shield, clean nonsterile gloves, and clear isolation gowns. All PPE should be donned before entering patient care area, must remain in place and worn correctly and must be removed slowly and deliberately in sequence to prevent self-contamination [18]. In this study, $84.9 \%$ of participants has a good knowledge on the use of appropriate PPE when dealing with patients with COVID-19. No previous study done to evaluate the PPE knowledge and preparedness in medical students, however, a recent international survey discussed this issue in neurosurgical residents and found that receiving formal training of the correct sequence for donning and doffing PPE was not sufficient [18].

Two thirds of the student are aware of the current Ministry of Health policies and recommendation on dealing with patients with COVID-19. Despite the emerging evidence on the importance of preparation and training for preparedness and management of patients with COVID-19, the study found a low levels of formal teaching and selflearning on management of patients with COVID-19 (18.2\% and $12.1 \%$ respectively). Inadequate career preparation has been identified as one of the core stressors for medical students [8].

In the Sultanate, on- campus classes in Higher Education Institutions have been suspended from the 15th March 2020 till further notice. The process of continued learning for these students has been affected. Both medical schools in Oman have relegated medical education to virtual learning platform. Alhaj et al reported that the number of daily studying hours was affected in approximately $80 \%$ of respondents. The same study revealed a significant impact of this pandemic on the mental health of $90 \%$ of the participants [18].

Several factors have been reported in influencing decisions on future specialities among senior medical students [19]. The challenges of the current pandemic prompted an emergent response and highlighted the role of Public Health and the WHO response in tackling such situation. In this study, about half of participants have agreed that the response to the pandemic has increased their interest in Public Health as a future career. The desire to work in challenging environment could be the reason for this motivation. This positive outcome of the pandemic or the influence on future career was not previously explored.

\section{CONCLUSION}

Graduating medical students join the medical taskforce with knowledge and experience gained throughout the undergraduate years. 'With ongoing rise in confirmed cases every day, the uncertainty of the duration of the suspension of on-campus teaching in higher education institutions in Oman is still ongoing. The COVID-19 pandemic has affected the teaching process and limited student- patient interactions.

The overall perceptions on preparedness for internship among medical students in Oman is good. However, further education in emergency management and training on practical ward procedures are still required. This study highlighted that a good effort in delivering the specific knowledge and practices on COVID-19 and patient management is recommended. Overall, there is satisfactory level of knowledge on COVID-19 between participants, however, gaps in knowledge related to hand hygiene and measures for infection control have been identified. The global response to COVID-19 pandemic has increased participants' interest in Public Health and Infection Control as a career.

\section{ACKNOWLEDGMENT}

Authors would like to thank the Gulf Research Collaboration Group (GRCG) for facilitating the distribution of the survey and providing support throughout the study period.

\section{REFERENCES}

[1] D. Wang, B. Hu, C. Hu, F. Zhu, X. Liu, J. Zhang, B. Wang, H. Xiang, Z. Cheng, Y. Xiong, Y. Zhao, Y. Li, X. Wang, Z. Peng, "Clinical characteristics of 138 hospitalized patients with 2019 novel coronavirus-infected pneumonia in Wuhan, China”, JAMA, vol. 323(11), pp. 1061-1069, 2020.

[2] WHO. (2020). Timeline-COVID-19. [Online]. Available: https://www.who.int/news-room/detail/27-04-2020-who-timeline--covid-19.

[3] D. Zou (March 2020). COVID-19: Implications for Medical student. Available: $\quad$ https://insightplus.mja.com.au/2020/12/covid-19implications-for-medical-students/

[4] A. People. (April 2020). Medical Students Can give vital help in the COVID-19 Crisis. Scientific American. [Online]. Available: https://blogs.scientificamerican.com/observations/medical-studentscan-give-vital-help-in-the-covid-19-crisis/

[5] Hospital emergency response checklist, World Health Organization regional office for Europe, 2011.

[6] P. A. Giri, G. S. Parhar, "Internship: A transition from medical student to A Doctor", International Journal of Biomedical and Advance Research, vol. 3, no 10, pp.753-755, 2012.

[7] S. R. Daugherty, D. C. Baldwin Jr, B. D. Rowley, "Learning, satisfaction and Mistreatment during medical internship: A National Survey of Working Conditions, vol. 279 (15), pp. 1194-1199, 1998.

[8] D. S. Eley, "Postgraduates' perceptions of preparedness for work as a doctor and making future career decisions: support for rural, nontraditional medical schools", Educ Health, vol. 23(2), p. 374, 2010.

[9] Information for healthcare professionals. (2020). [Online]. Available: https://www.cdc.gov/coronavirus/2019-ncov/hcp/index.html.

[10] WHO. (2020). Coronavirus Disease (Covid-19) Outbreak: Rights, Roles and Responsibilities of Health Workers, Including Key Considerations for Occupational Safety and Health. [Online]. 
[11] WHO collaboration in Oman's response to COVID-19. [Online] Available: $\quad$ http://www.emro.who.int/omn/oman-news/whocollaboration-on-omans-response-to-covid-19.html.

[12] MOH Statement No (72) 08 of May 2020. Ministry of Health, Sultanate Oman. [Online]. Available: https://www.moh.gov.om/en/-/72-8-2020-154-

[13] A. Harvey, "Covid-19: medical schools given powers to graduate final year students early to help NHS", BMJ, vol. 368:m1227, 2020.

[14] Q. Li, X. Guan, P. Wu, X. Wang, L. Zhou, Y. Tong, et al. "Early Transmission Dynamics in Wuhan, China, of Novel CoronavirusInfected Pneumonia", N Engl J Med, vol. 382, no. 13, pp. 1199-1207, 2020.

[15] P. D. Modi, G. Nair, A. Uppe, et al. "COVID-19 Awareness Among Healthcare Students and Professionals in Mumbai Metropolitan Region: A Questionnaire-Based Survey", Cureus, vol. 12(4), 2020.

[16] A. J. Alsahafi, A. C. Cheng, "Knowledge, attitudes and behaviors of healthcare workers in the Kingdom of Saudi Arabia to MERS coronavirus and other emerging infectious diseases", Int J Environ Res Public Health, vol. 13, 2016.

[17] Coronavirus Disease 2019 (COVID-19). [Online]. Available: https://www.cdc.gov/coronavirus/2019-ncov/hcp/hand-hygiene.html.

[18] A. Kh. Alhaj, T. Al-Saadi, F. Mohammad, and S. Alabri, "Neurosurgery Residents Perspective on the COVID-19: Knowledge, Readiness, and Impact of this Pandemic", World Neurosurgery, [published online ahead of print, 2020 May 16], 2020.

[19] Interim infection prevention and control recommendations for patient with confirmed 2019 novel coronavirus (2019-nCoV) or patients under investigation for $2019-\mathrm{nCoV}$ in healthcare settings. Centers for Disease Control and Prevention. [Online]. Available: https://www.cdc.gov/coronavirus/2019-nCoV/hcp/infectioncontrol.html.

[20] R. Al-Fouzan, S. Al-Ajlan, Y. Marwan, M. Al-Saleh, "Factors affecting future specialty choice among medical students in Kuwait", Med Educ Online, vol. 17, pp. 1-7, 2012. 